\title{
Expressive timing, musical tension, and listener-performer synchronicity: Commentary on Ohriner
}

\author{
BRUNO GINGRAS [1] \\ University of Vienna, Vienna, Austria
}

\begin{abstract}
Ohriner (this volume) empirically investigated the ability of listeners to rhythmically entrain to performances of Chopin's mazurkas and suggested that performers can manipulate listeners' expectations and influence their perceived musical tension by using eccentric or unpredictable patterns of tempo variation. In this commentary, I attempt to situate Ohriner's research in a broader context, while elaborating on his findings and proposing alternative interpretations in some cases. I suggest that, although mazurkas are particularly suitable for a study of entrainment due to their clear metrical structure, it would be appropriate to study musical genres in which the metrical structure is obscure or even absent. I also question Ohriner's interpretation of a lack of synchronicity as being predominantly associated with negative emotions. Furthermore, in order to examine more rigorously the influence of synchronicity on perceived musical tension, I present a controlled experimental design to disentangle the effects of tempo and rubato, which would involve measuring both entrainment and musical tension while asking participants to rate the perceived eccentricity of the performances.
\end{abstract}

Submitted 2014 July 28; accepted 2014 August 31.

KEYWORDS: performance, synchronization, expressive timing, expectations, musical tension

IN an empirical study published in this issue, Ohriner investigated the ability of listeners to rhythmically entrain to musical performances exhibiting large tempo fluctuations, by asking them to tap along to two selected passages from Chopin's mazurkas. According to Ohriner, the "predictability" of expressive timing in performance should itself be considered as an expressive strategy that can be used to modulate the listener's emotions. This is a plausible idea, given that melodic and harmonic expectations, and the ways in which composers manipulate them, have been shown to affect the perception of musical tension (Huron, 2006; Krumhansl, 1996, 2002; Meyer, 1956). Moreover, Ohriner's experiment incorporates several elements that have been at the forefront of recent research on rhythm perception. Notably, it has been shown that participants tapping along with the beat were more accurate in predicting the timing of a final tone than participants listening without moving (Manning \& Schultz, 2013) and, more generally, that body movement enhances auditory temporal sensitivity (Su \& Pöppel, 2011).

\section{RUBATO AND THE COMMUNICATIVE PRESSURE PRINCIPLE}

The study focuses exclusively on Chopin's mazurkas. This is a valid starting point, given that Chopin's music is widely viewed as being particularly suited for rubato in performance (Matthay, 1913; Stein, 1962). Temperley's (2004) communicative pressure principle predicts a tradeoff between the amount of rubato (or more generally tempo variation in performance) on the one hand, and the clarity of the metrical structure of a piece on the other hand. Thus, musical contexts in which the meter is strongly reinforced should provide greater opportunities for tempo variation, given that the underlying beat remains easy to follow. Indeed, Chopin's mazurkas typically exhibit a very standard rhythmic and metrical framework and commonly use repeated melodic patterns, especially in the left hand, which are an important cue to meter (Steedman, 1977; Temperley \& Bartlette, 2002). In that sense, the eccentric renditions selected by Ohriner provide an interesting test of Temperley's hypothesis: how much rubato can performers apply before listeners are 
unable to synchronize or to feel a sense of pulse (Madison \& Merker, 2002), in a repertoire characterized by a transparent metrical structure?

At the same time, if the goal is to explore specifically the synchronization between performer and listener, it would also be appropriate to study musical genres in which the metrical structure is more obscure or even absent, as mentioned briefly by Ohriner in his concluding remarks. The unmeasured prelude, a genre associated with French harpsichord music of the $17^{\text {th }}$ and early $18^{\text {th }}$ centuries and characterized by the absence of meter and, in some cases, of notated durations, would seem particularly suitable for such an extension of Ohriner's study, especially in cases for which the exact performance timings and perceived musical tension ratings are available (Gingras, Pearce, Goodchild, Dean, Wiggins, \& McAdams, submitted; Goodchild, Gingras, \& McAdams, submitted).

\section{SYNCHRONICITY, MUSICAL TENSION, AND FAMILIARITY}

Following Huron (2006), Ohriner posits that synchronization with a musical performance is positively valenced, whereas an inability to synchronize may lead to a negatively valenced state. Although he nuances this statement by noting that asynchrony is not necessarily detrimental, the author gives perhaps too much credence to the idea that an inability to synchronize is associated with negative emotional connotations. Huron (2006) himself notes that less predictable events such as syncopations, hemiolas, and missing downbeats can also evoke positive emotions (p. 185). Moreover, in the case of the unmeasured prelude for instance, it is not clear that listeners would feel the need to tap along with a performance (as may be the case for music with a strong, regular pulse), or even that they could do it accurately. However, this does not necessarily imply that listening to unmeasured music, for which a lack of listener-performer synchronicity is to be expected, is an unpleasant experience.

Along the same lines, Ohriner sometimes appears to suggest that synchronicity between performer and listener is a major determinant of musical tension and emotion (see for instance the section on "Listener-performer synchronicity in an altered return"). In my opinion, this view may overstate the importance of synchronicity. Perhaps a controlled experiment using synthesized performances varying only in rubato (with the same mean tempo, sound intensity, etc...), in which participants are asked to continuously rate their perceived musical tension in addition to tapping along with the performances (the two tasks being conducted in successive listenings, for instance), would be a way to compare more specifically tapping asynchrony and perceived musical tension. This would also have the advantage of allowing the experimenter to disentangle between the effects related to tempo itself and those due to variations in tempo.

Ohriner specifically sought performances that exhibited unique choices of tempo variations among a collection of many renditions of Chopin's mazurkas. However, it is difficult to estimate to what extent listeners were aware of the uniqueness or idiosyncrasy of these performances. Listeners' familiarity, both with the repertoire and with performances of the Chopin mazurkas chosen here, may influence their ability to synchronize with these renditions, as well as their ability to recognize their eccentricity. Ohriner, citing London's (2004) "Many Meters Hypothesis", acknowledges the need to evaluate participants' familiarity with Chopin's style. I would also suggest asking participants to rate the uniqueness or eccentricity of the performances they heard, and evaluating whether these ratings correlated with the synchronization accuracy. This would be particularly relevant in light of the observation that more idiosyncratic performances, such as Roberto Poli's recording of Op. 50, No. 1, were apparently more difficult to tap along.

Two points mentioned by Ohriner regarding the asymmetry between late and early taps deserve further discussion. First, complementing Ohriner's point regarding the distinction between late and early taps, listeners have been shown to be more accurate in detecting late versus early events (Large \& Jones, 1999; Manning \& Schultz, 2013), while musicians were found to be better at coordinating with decreasing rather than increasing tempi (Loehr, Large, \& Palmer, 2011). Second, the author rightly points out that agreement between performer and listener on the direction of tempo change (acceleration or deceleration), even in cases where the listener underestimates a deceleration, is quite different from an inaccurate tap in the opposite direction of the actual tempo change. This suggests that, instead of analyzing the deviation between taps and beat onsets in performance, it might be more appropriate to evaluate the correlation between inter-beat intervals and inter-tap intervals. Ohriner, citing Repp (2002), discusses this idea but considers that it is not appropriate for musical performances due to the influence of the musical context. While this may be a valid point, it leads to an analysis prioritizing tapping accuracy over agreement 
between performer and listener, although the latter may be more relevant in the case of performances with extensive tempo variations.

\section{CONCLUSION}

In his study, Ohriner presents a promising method for investigating the musical communication between performer and listener, especially regarding the link between rubato and the melodic and harmonic expectations generated by the musical structure. More broadly, this work can be seen as an extension of more fundamental work investigating the connection between expectations in pitch space and temporal expectations (Firmino, Bueno, \& Bigand, 2009) or the influence of unexpected melodic events on temporal expectations (Bailes, Dean, \& Pearce, 2013). Indeed, Ohriner makes some tantalizing observations about the connection between harmonic and temporal expectations, and more specifically about the performer's role in modulating these expectations, which call for further exploration.

At the same time, this work can be developed further, and perhaps in a more rigorous direction, by explicitly isolating the effects of tempo on performer-listener synchronicity (and accessorily perceived musical tension) from those of tempo variations. It would also be worthwhile to apply this methodology to

a wider musical repertoire, and in a manner that takes into account the influence of listeners' familiarity with a musical style on their ability to tap along. In sum, Ohriner's study is an auspicious first step towards the development of a new field of empirical inquiry in music performance research.

\section{NOTES}

[1] Correspondence concerning this commentary should be addressed to: Dr. Bruno Gingras, Department of Cognitive Biology, Faculty of Life Sciences, University of Vienna, Vienna, Austria A-1090. Email: brunogingras@gmail.com

\section{REFERENCES}

Bailes, F., Dean, R. T., \& Pearce, M. T. (2013). Music cognition as mental time travel. Scientific Reports, $3,2690$.

Firmino E. A., Bueno J. L. O., \& Bigand E. (2009). Travelling through pitch space speeds up musical time. Music Perception, 26(3), 205-209.

Gingras, B., Pearce, M. T., Goodchild, M., Dean, R. T., Wiggins, G., \& McAdams, S. (under review). Linking melodic expectation to expressive performance timing and perceived musical tension.

Goodchild, M., Gingras, B., \& McAdams, S. (under review). Analysis, performance, and tension perception of an unmeasured prelude for harpsichord.

Huron, D. B. (2006). Sweet anticipation: Music and the psychology of expectation. Cambridge, MA: MIT Press.

Iordanescu, L., Grabowecky, M., \& Suzuki, S. (2013). Action enhances auditory but not visual temporal sensitivity. Psychonomic Bulletin and Review, 20, 108-114.

Krumhansl, C. L. (1996). A perceptual analysis of Mozart's Piano Sonata K.282: Segmentation, tension, and musical ideas. Music Perception, 13(3), 401-432.

Krumhans1, C. L. (2002). Music: A link between cognition and emotion. Current Directions in Psychological Science, 11(2), 45-50.

Large, E. W., \& Jones, M. R. (1999). The dynamics of attending: How people track time-varying events. Psychological Review, 106(1), 119-159. 
Loehr, J. D., Large, E. W., \& Palmer, C. (2011). Temporal coordination and adaptation to rate change in music performance. Journal of Experimental Psychology: Human Perception and Performance, 37, 12921309.

London, J. (2004). Hearing in time: Psychological aspects of musical meter. Oxford: Oxford University Press.

Madison, G., \& Merker, B. (2002). On the limits of anisochrony in pulse attribution. Psychological Research, 66(3), 201-207.

Manning, F., \& Schultz, M. (2013). "Moving to the beat" improves timing perception. Psychonomic Bulletin and Review, 20, 1133-1139.

Matthay, T. (1913). Musical interpretation. London: Joseph Williams Ltd.

Meyer, L. B. (1956). Emotion and meaning in music. Chicago: University of Chicago Press.

Repp, B. H. (2002). The embodiment of musical structure: Effects of musical context on sensorimotor synchronization with complex timing patterns. In: W. Prinz \& B. Hommel (Eds.), Common Mechanisms in Perception and Action: Attention and Performance XIX (pp. 245-265). New York: Oxford University Press.

Steedman, M. (1977). The perception of musical rhythm and meter. Perception, 6, 555-570.

Stein, E. (1962/1989). Form and performance. New York: Limelight Editions.

Su, Y.-H., \& Pöppel, E. (2011). Body movement enhances the extraction of temporal structures in auditory sequences. Psychological Research, 76(3), 373-382.

Temperley, D. (2004). Communicative pressure and the evolution of musical styles. Music Perception, 21(3), 313-337.

Temperley, D., \& Bartlette, C. (2002). Parallelism as a factor in metrical analysis. Music Perception, 20, 117-149. 